\title{
Single-Cycle Impulse from Detonation Tubes with Nozzles
}

\author{
M. Cooper* \\ Sandia National Laboratories, Albuquerque, New Mexico 87185 \\ and \\ J. E. Shepherd \\ California Institute of Technology, Pasadena, California 91125 \\ DOI: $10.2514 / 1.30192$
}

\begin{abstract}
Experiments measuring the single-cycle impulse from detonation tubes with nozzles were conducted by hanging the tubes in a ballistic pendulum arrangement within a large tank. The detonation-tube nozzle and surrounding tank were initially filled with air between 1.4 and $100 \mathrm{kPa}$ in pressure simulating high-altitude conditions. A stoichiometric ethylene-oxygen mixture at an initial pressure of $80 \mathrm{kPa}$ filled the constant-diameter portion of the tube. Four diverging nozzles and six converging-diverging nozzles were tested. Two regimes of nozzle operation were identified, depending on the environmental pressure. Near sea-level conditions, unsteady gas-dynamic effects associated with the mass of air contained in the nozzle increase the impulse as much as $72 \%$ for the largest nozzle tested over the baseline case of a plain tube. Near vacuum conditions, the nozzles quasi-steadily expand the flow, increasing the impulse as much as $43 \%$ for the largest nozzle tested over the baseline case of a plain tube. Competition between the unsteady and quasi-steady-flow processes in the nozzle determine the measured impulse as the environmental pressure varies.
\end{abstract}

\section{Nomenclature}

$A$
$C$
$D$
$F$
$g$
$h$
$I$
$I_{\mathrm{sp}}$
$I_{\mathrm{sp}}^{0}$
$K$
$L$
$\mathcal{M}$
$\dot{m}$
$N$
$P$
$T$
$t$
$U_{\mathrm{CJ}}$
$\bar{u}$
$u$
$V$
$\mathbf{Y}$
$\rho$
$\phi$

$=$ area

$=$ combustible mixture mass

$=$ diameter

$=$ force

$=$ gravitational acceleration

$=$ enthalpy per unit mass

$=$ impulse

$=$ mixture-based specific impulse

mixture-based specific impulse of the plain tube

model proportionality constant

tube length

mass

- mass flow rate

tamper mass

pressure

$=$ temperature

time

= Chapman-Jouguet detonation velocity

$=$ average velocity

$=$ velocity

$=$ volume

$=$ species concentration

$=$ density

$=$ nozzle half-angle

Subscripts

$C=$ converging portion of the nozzle

$D=$ diverging portion of the nozzle

$i=$ nozzle inlet state

throat $=$ state at the throat of the converging-diverging nozzle

$\mathrm{TS}=$ thrust surface

Received 1 February 2007; revision received 10 August 2007; accepted for publication 22 August 2007. Copyright (C) 2007 by California Institute of Technology. Published by the American Institute of Aeronautics and Astronautics, Inc., with permission. Copies of this paper may be made for personal or internal use, on condition that the copier pay the $\$ 10.00$ per-copy fee to the Copyright Clearance Center, Inc., 222 Rosewood Drive, Danvers, MA 01923; include the code 0748-4658/08 \$10.00 in correspondence with the CCC.

*Senior Member of Technical Staff, Explosives Technologies Group.

${ }^{\dagger}$ Professor. Member AIAA.

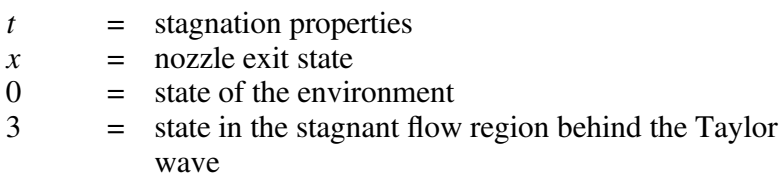

\section{Introduction}

N INCREASE in single-cycle impulse is realized when a A diverging nozzle is added to the open end of a detonation tube, making nozzles attractive to detonation engine developers. Much of the previous research on detonation-tube nozzles has focused on single-cycle numerical simulations [ $\underline{1}-\underline{6}]$ or experiments conducted at atmospheric environmental pressures [7-10]. Additional studies on nozzles are reviewed by Morris [5], Kailasanth [11], and Allgood et al. [7]. The goal of this work was to characterize nozzle performance on single-cycle detonation tubes for a variety of nozzle pressure ratios expected at realistic operating conditions. Depending on the surrounding environmental pressure, the nozzle acts to increase the impulse through either unsteady or quasi-steady gas dynamics. This work presents experimental single-cycle impulse data for different pressure ratios, illustrating the two observed regimes of nozzle operation.

Careful consideration is required before extending the conclusions of this paper to multicycle detonation tubes. As demonstrated by a number of experimental $[7,12]$ and numerical $[1,13-15]$ studies, the filling and purging portions of a cycle can significantly affect the nozzle flow processes. In our tests, the environmental gas contained within the nozzle is initially at rest at the environmental gas pressure. The environmental gas must be accelerated out of the nozzle after the diaphragm at the interface between the constant-diameter portion of the tube and the nozzle inlet bursts. Alternatively, in a multicycle device, the gases within the nozzle remain in motion due to the purging and filling processes. This difference in the initial gas velocity within the nozzle at the time when the detonation products enter the nozzle will directly affect the measured impulse. Although this paper will not address many of the issues faced by multicycle pulse detonation engine nozzle designers, the experiments do highlight the critical factors affecting nozzle performance on singlecycle detonation tubes.

This paper complements previous work determining the singlecycle impulse of fully filled constant-diameter detonation tubes at subatmospheric environments [16,17]. Mixtures of stoichiometric ethylene-oxygen were detonated in a tube that exhausted into air 


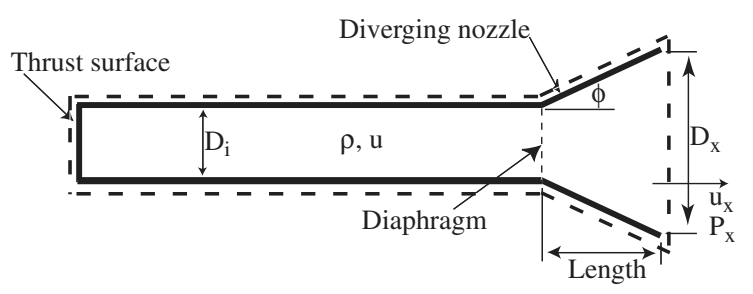

Fig. 1 Illustration of the detonation tube with a diverging nozzle.

between 1.4 and $100 \mathrm{kPa}$ in pressure. The specific impulse was found to increase as the environmental pressure decreased and was predicted with the original impulse model of Wintenberger et al. [18] modified to account for the increased blowdown time to the lower environmental pressures. In the following sections, single-cycle impulse data are presented for detonation tubes with diverging and converging-diverging nozzles. The effects of nozzle area ratio, explosive mass fraction, and nozzle length are investigated. To understand the two regimes of nozzle operation, comparisons are made to impulse predictions using steady- and unsteady-flow assumptions.

\section{Experimental Facility}

Experiments were carried out in a 1.014-m-long, 76.2-mm-diam detonation tube. One end of the tube was closed, forming the thrust surface. A nozzle was attached to the other end of the tube. A stoichiometric ethylene-oxygen mixture at a pressure of $P_{1}=$ $80 \mathrm{kPa}$ initially filled the constant-diameter tube and was prevented from entering the nozzle by a $105-\mu \mathrm{m}$-thick Mylar® diaphragm, as illustrated in Fig. 1. The tube was outfitted with pressure transducers and ionization gauges and hung in a ballistic pendulum arrangement within a 12,500-liter pressure vessel [16,17]. The volume inside the large pressure vessel was filled with room air at environmental pressures $P_{0}$ between 1.4 and $100 \mathrm{kPa}$. Detonations were initiated within $1 \mathrm{~cm}$ of the thrust surface by deflagration-to-detonation transition. A standard aircraft spark plug with 30-mJ discharge energy initiated the deflagration. The maximum deflection of the tube was converted into impulse by applying the classical analysis of an impulsively created motion and the conservation of energy [8]. Measured deflections observed in our experiments were between 39 and $292 \mathrm{~mm}$. The experimental uncertainty in the specific impulse was estimated to be $\pm 3.8 \%$ [8].

The four conical diverging nozzles tested are listed in Table 1 and illustrated in Fig. 1. A straight extension is listed in the table and, for convenience, is described in this paper as a diverging nozzle with an area ratio of 1 and a half-angle $\phi$ of 0 .

\section{Diverging Nozzle Results}

The specific impulse data are plotted in Fig. 2 as a function of the environmental pressure for a plain tube (no nozzle) and for tubes with 8- and 12-deg nozzles. Refer to Table 2 for tabulated data of the percent difference between the measured impulse with a nozzle $I_{\mathrm{sp}}$ and the impulse of a plain tube $I_{\mathrm{sp}}^{0}$ at each environmental pressure. The impulse is shown to always increase at a given environmental pressure by adding a diverging nozzle onto a plain tube. Consider the case of a plain tube: The impulse is shown to always increase with decreasing the environmental pressure, due to a longer blowdown time and an increased pressure differential across the thrust surface [17]. For the case of a tube with a diverging nozzle, the impulse may

Table 1 Dimensions of the diverging nozzles

\begin{tabular}{lccccc}
\hline \hline Description & Length, m & $\phi, \mathrm{deg}$ & $D_{i}, \mathrm{~mm}$ & $D_{x}, \mathrm{~mm}$ & $A_{x} / A_{i}$ \\
\hline $0 \mathrm{deg}, 0.6 \mathrm{~m}$ & 0.6 & 0 & 63.5 & 63.5 & 1 \\
$8 \mathrm{deg}, 0.3 \mathrm{~m}$ & 0.3 & 8 & 63.5 & 152.0 & 5.7 \\
$12 \mathrm{deg}, 0.3 \mathrm{~m}$ & 0.3 & 12 & 76.2 & 194.0 & 6.5 \\
$12 \mathrm{deg}, 0.6 \mathrm{~m}$ & 0.6 & 12 & 76.2 & 311.0 & 16.7 \\
\hline \hline
\end{tabular}

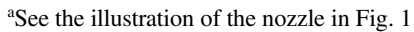

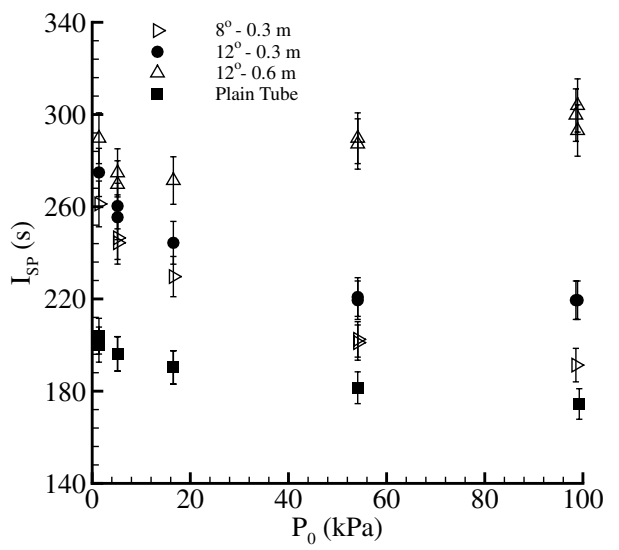

Fig. 2 Specific impulse as a function of environmental pressure for detonation tubes with diverging nozzles.

increase or decrease with changes in the environmental pressure. To understand the effect on the impulse of a diverging nozzle, the assumptions of quasi-steady and unsteady flow are considered individually.

The quasi-steady-flow analysis is based on the standard assumptions of a steady-flow rocket and appears in Sec. IV. Central to the unsteady-flow analysis given in Sec. $\mathrm{V}$ is the Gurney model [19], which uses the tamper mass ratio $N / C$ and the explosive mass fraction $C /(N+C)$. Values for these parameters for each nozzle tested appear in Table 2. The Gurney model uses an asymmetric sandwich consisting of the tube mass, the explosive mixture mass $C$ contained in the constant-diameter portion of the tube, and the tamper mass $N$ contained in the nozzle. The tube mass-explosive-tamper mass sandwich is considered to be asymmetrical because $N$ is always less than the mass of the tube. Detonation of the explosive mixture imparts linear momentum to both the tube mass and the tamper mass based on momentum and energy conservation within the sandwich. A linear velocity gradient within the explosive products is assumed, propelling the tube and tamper masses in opposite directions. Thus, as the tamper mass becomes large compared with the tube mass, more of the explosive mixture energy is directed into accelerating the tube mass. This is a one-dimensional idealized model used to formulate the partial-fill model [16], and so the diverging nozzles are considered to be one-dimensional extensions with an equivalent tamper mass.

The impulse of a tube increases with increasing tamper. This is shown in Fig. 2 by comparing the impulses from the diverging nozzles at a constant environmental pressure. For example, at an environmental pressure of $100 \mathrm{kPa}$, the $12-\mathrm{deg}, 0.6-\mathrm{m}$ nozzle has the largest value of $N / C$ and the greatest impulse. Decreasing the tamper by decreasing the nozzle volume (e.g., decreasing length or halfangle) results in a corresponding decrease in impulse. When the impulse depends significantly on the unsteady gas dynamics of the tamper mass, the nozzle is operating in the unsteady regime.

When the impulse depends on quasi-steady-flow expansion at the lowest environmental pressures, the nozzle is operating in the quasisteady regime. The tamper mass fraction is nearly zero and the environmental pressure is sufficiently low so that expansion of the detonation products is not restricted. Again, the 12-deg, 0.6-m nozzle generates the largest increase in impulse of $43 \%$ over the baseline case of a plain tube at an environmental pressure of $1.4 \mathrm{kPa}$. From this value, decreasing the nozzle area ratio reduces the amount of flow expansion (and impulse). Thus, the nozzle area ratio dominates the impulse. For the 12-deg, 0.6-m nozzle, it is interesting to note that the impulse in the unsteady-flow regime is actually greater than the impulse in the quasi-steady-flow regime.

Inspecting the impulse of a specific nozzle as the environmental pressure decreases illustrates the shift between the unsteady and quasi-steady operating regimes. A transition environmental pressure exists in which neither the unsteady nor the quasi-steady-flow regimes dominate the measured impulse. This transition occurs at the environmental pressure associated with a minimum impulse. As the 
Table 2 Percent increases in specific impulse for the diverging nozzles

\begin{tabular}{|c|c|c|c|c|}
\hline & $P_{0}, \mathrm{kPa}$ & Tamper mass ratio $N / C$ & Explosive mass fraction $C /(N+C)$ & $\left(I_{\mathrm{sp}}-I_{\mathrm{sp}}^{0}\right) / I_{\mathrm{sp}}^{0}$ measured from Figs. $\underline{2}$ and $\underline{3}, \%$ \\
\hline \multirow[t]{5}{*}{$0 \mathrm{deg}, 0.6 \mathrm{~m}$} & 100 & 0.73 & 0.58 & 26 \\
\hline & 54.5 & 0.39 & 0.72 & 16 \\
\hline & 16.5 & 0.12 & 0.89 & 10 \\
\hline & 5.2 & 0.04 & 0.96 & 9 \\
\hline & 1.4 & 0.01 & 0.99 & 13 \\
\hline \multirow[t]{5}{*}{$8 \mathrm{deg}, 0.3 \mathrm{~m}$} & 100 & 0.65 & 0.61 & 6.4 \\
\hline & 54.5 & 0.41 & 0.71 & 10 \\
\hline & 16.5 & 0.13 & 0.89 & 20 \\
\hline & 5.2 & 0.038 & 0.96 & 25 \\
\hline & 1.4 & 0.016 & 0.99 & 29 \\
\hline \multirow[t]{5}{*}{$12 \mathrm{deg}, 0.3 \mathrm{~m}$} & 100 & 1.17 & 0.46 & 26 \\
\hline & 54.5 & 0.63 & 0.61 & 22 \\
\hline & 16.5 & 0.20 & 0.83 & 28 \\
\hline & 5.2 & 0.058 & 0.94 & 31 \\
\hline & 1.4 & 0.016 & 0.98 & 36 \\
\hline \multirow[t]{5}{*}{$12 \mathrm{deg}, 0.6 \mathrm{~m}$} & 100 & 5.0 & 0.17 & 72 \\
\hline & 54.5 & 2.7 & 0.27 & 59 \\
\hline & 16.5 & 0.9 & 0.54 & 43 \\
\hline & 5.2 & 0.3 & 0.80 & 39 \\
\hline & 1.4 & 0.1 & 0.93 & 43 \\
\hline
\end{tabular}

environmental pressure decreases, the impulse decreases in the unsteady-flow regime due to a decrease in tamper mass. The impulse decreases to a minimum before the quasi-steady flow begins to dominate and flow expansion to the lowest environmental pressures occurs. Because the changes in tamper mass and flow expansion as a function of the environmental pressure depend on the nozzle shape, a different transition environmental pressure exists for each nozzle. The 12-deg, 0.6-m nozzle transitions between operating regimes at an environmental pressure of $5.2 \mathrm{kPa}$ and the 12-deg, 0.3-m nozzle transitions at a pressure of $54.5 \mathrm{kPa}$. The $8-\mathrm{deg}, 0.3-\mathrm{m}$ nozzle appears to be always within the quasi-steady-flow regime as the impulse increases for all decreases in environmental pressure.

The effect of nozzle shape on impulse is observed in Fig. 3 by comparing impulses of the 0-deg, 0.6- $\mathrm{m}$ and 8-deg, 0.3-m nozzles. Although the 8-deg, 0.3-m nozzle has half the length of the straight extension, their explosive and tamper mass fractions are nearly identical over the range of tested environmental pressures (Table 2). The mass-based partial-fill model [16] therefore predicts that both nozzles should yield the same impulses. Instead, a $6.4 \%$ increase in impulse over a plain tube is observed with the 8-deg, 0.3-m nozzle, whereas the 0-deg, 0.6-m nozzle observes a $26 \%$ increase over a plain tube at an environmental pressure of $100 \mathrm{kPa}$. This illustrates that in the unsteady-flow regime, the effect of the tamper mass is more efficient at increasing the impulse in one-dimensional geometries. At an environmental pressure of $1.4 \mathrm{kPa}$, the impulse of the 8 -deg, $0.3-\mathrm{m}$ nozzle increases $29 \%$ over a plain tube and $16 \%$ over the 0-deg, 0.6-m nozzle. At this low $P_{0}$, the diverging nozzle generates higher values of impulse due to the additional flow

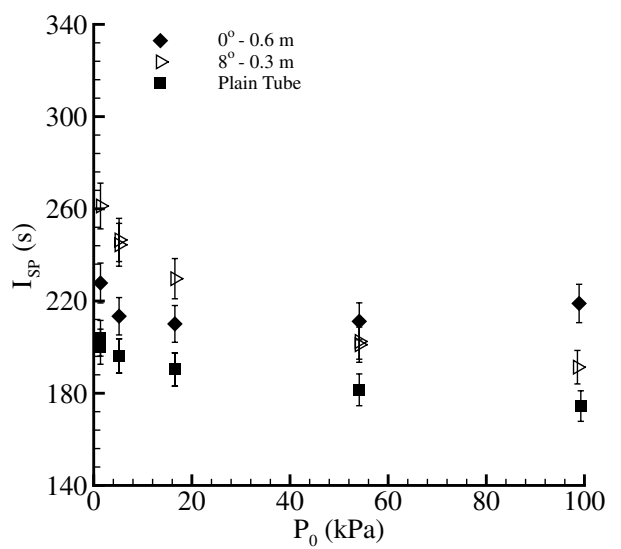

Fig. 3 Specific impulse as a function of environmental pressure for detonation tubes with the straight extension and the 8-deg, 0.3-m nozzle. expansion provided by the divergent shape. Clearly, in the quasisteady regime, the nozzle area ratio dominates.

\section{Quasi-Steady-Flow Regime}

The nozzle performance at the lowest environmental pressures can be qualitatively modeled using the steady-flow rocket equation. Consider a control volume that surrounds the detonation tube and nozzle, as shown in Fig. 1. The general unsteady mass conservation for the control volume is

$$
\frac{\mathrm{d} \mathcal{M}}{\mathrm{d} t}+\dot{m}(t)=0
$$

and the general unsteady momentum conservation for the control volume consists of the pressure forces and the exhaust gas momentum.

$$
F(t)=\dot{m}(t) u_{x}(t)+A_{x}\left[P_{x}(t)-P_{0}\right]+\frac{\mathrm{d}}{\mathrm{d} t} \int_{V} \rho u \mathrm{~d} V
$$

The second term on the right-hand side of Eq. (2) is zero for pressurematched conditions at the nozzle exit. The third term on the righthand side of Eq. (2) corresponds to the unsteady variation of momentum inside the control volume. This term is typically neglected when analyzing steady rocket engines [20]. Within a detonation tube, the detonation wave and Taylor wave reflection off the area change at the nozzle inlet increase the momentum, whereas the Taylor wave decreases the gas momentum to zero. Thus, although the unsteady variation of momentum inside the detonationtube control volume is greater than zero, for the sake of modeling nozzle performance, it is assumed to be negligible.

With these simplifications, the specific impulse can be written as

$$
I_{\mathrm{sp}}=\frac{\int F(t) \mathrm{d} t}{\int \dot{m}(t) g \mathrm{~d} t}=\frac{\int \dot{m}(t) u_{x}(t) \mathrm{d} t}{\int \dot{m}(t) g \mathrm{~d} t}
$$

The nozzle exit velocity and mass flow are constant in time in the case of a steady-flow rocket engine, and so Eq. (3) reduces to

$$
I_{\mathrm{sp}}=\frac{u_{x}}{g}
$$

The nozzle exit velocity and mass flow are not constant in time in the case of a detonation tube, and so for simplicity, an average exit velocity $\bar{u}_{x}$ is assumed. Replacing $u_{x}(t)$ with $\bar{u}_{x}$ in Eq. (3) prevents determination of the time-varying mass flow out of the tube and allows $\bar{u}_{x}$ to be calculated from the measured $I_{\mathrm{sp}}$ data. 


$$
\bar{u}_{x}=I_{\mathrm{sp}} g
$$

Comparing the steady-flow predictions of $u_{x}$ and $I_{\mathrm{sp}}$ to the detonation-tube values of the calculated $\bar{u}_{x}$ and measured $I_{\mathrm{sp}}$ is not strictly valid, due to the inherent unsteadiness within the detonationtube flow. However, if we assume that any variations from the ideal steady-flow case are minor or can be reasonably modeled, then the impulse from a detonation tube with a nozzle can be compared with the impulse from an ideal steady-flow nozzle with the same dimensions. The merit of conducting this analysis is to generate an ultimate measure of performance for detonation tubes with nozzles.

Two processes that affect this modeling approach are the assumption of quasi-steady flow throughout most of the blowdown process and the assumption that the continuously decreasing pressure upstream of the nozzle inlet does not significantly affect the quasi-steady nozzle flow. The first assumption implies a rapid startup time and unchoking of the nozzle inlet late in the blowdown process. The second assumption implies that the continuous pressure decrease upstream of the nozzle inlet can be modeled or accounted for.

\section{A. Nozzle Startup Time}

The elapsed time from when the transmitted shock wave enters the nozzle until quasi-steady flow is established within the nozzle should be small compared with the cycle time to maximize the impulse. The presence of this transient nozzle flow has previously been studied in shock tunnels [21-23] and rocket nozzles [24].

For detonation-tube nozzle flows, the startup process is likely affected by the Taylor wave pressure profile that exists behind the shock wave just after it enters the nozzle inlet. For simplicity, an estimate of the detonation-tube nozzle startup time is made from the time taken by a particle as it travels under steady-flow conditions from the inlet to the exit of the nozzle. The startup time is assumed to equal three durations of this steady-flow time, which is then compared with the total single-cycle time of the detonation tube. The time duration, as determined from the measured pressure histories, from ignition to the end of the blowdown process is approximately $4000 \mu$ s for the 8-deg, 0.3-m nozzle, $4500 \mu$ s for the 12-deg, 0.3-m nozzle, and $5000 \mu \mathrm{s}$ for the 12-deg, 0.6- $\mathrm{m}$. Three durations of the steady-flow time determined from the previous finite rate calculations yield values of $252 \mu$ s for the 8-deg, 0.3-m nozzle, $354 \mu$ s for the 12-deg, 0.3-m nozzle, and $642 \mu$ s for the 12-deg, 0.6$\mathrm{m}$ nozzle. Thus, the startup time is expected to range between 6 and $12 \%$ of the total cycle time. Improved estimates of the nozzle startup time for the different environmental pressures are possible with experiments that enable visualization of the nozzle flowfield (such as those of Owens and Hanson [12]) or numerical simulations that include the effects of viscosity.

\section{B. Nozzle Inlet State}

The pressure upstream of the nozzle inlet on a detonation tube decreases in time, whereas the steady-flow modeling approach of Eq. (5) assumes a constant upstream pressure. Thus, the representative and constant nozzle inlet state for the detonation tube must be carefully chosen. Two choices for a representative upstream pressure consist of the pressure of the stationary gas at the thrust surface and behind the Taylor wave (referred to as state 3 ) or an average pressure based on a time average at a specific location within the constant-diameter portion of the detonation tube. An average pressure was determined by time-averaging the measured thrust surface pressure history in a plain tube for one cycle. This method yielded an average value of $400 \mathrm{kPa}$ (compared with the state-3 pressure of $970 \mathrm{kPa}$ ) for the ethylene-oxygen mixtures at an initial pressure of $80 \mathrm{kPa}$.

From the upstream pressure, the flow is assumed to isentropically expand to sonic conditions at the nozzle inlet so that the total enthalpy is conserved.

$$
u(P)=\sqrt{2\left[h_{3}-h(P)\right]}
$$

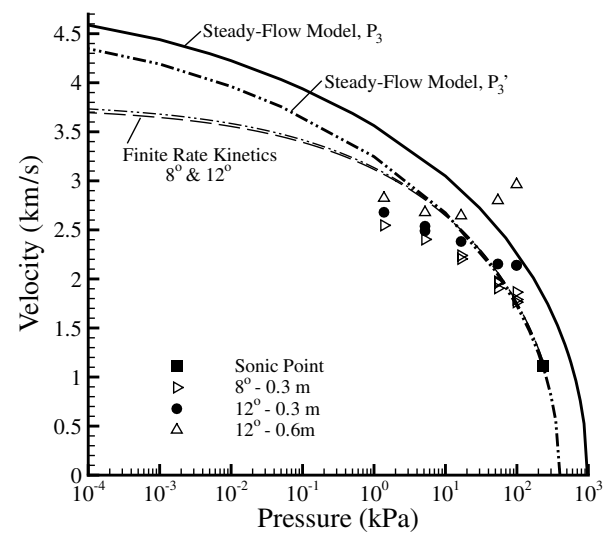

Fig. 4 Data and predictions of the nozzle exit velocity as a function of the environmental pressure starting from either the state 3 or an average upstream pressure. The calculations were conducted assuming either equilibrium composition or finite rate chemical kinetics.

From the sonic point, the flow is steadily expanded by the nozzle to the environmental pressure. A limiting velocity and specific impulse is predicted from the expansion to low pressures.

$$
u \rightarrow u_{\max }=\lim _{P \rightarrow 0} \sqrt{2\left[h_{t}-h(P)\right]}
$$

Because $h=h(\mathbf{Y}, T)$, species and temperature variations need to be related to the pressure variation to predict $h$. This can be done with calculations that assume either frozen or equilibrium chemical composition or finite rate chemical kinetics. In the case of steady supersonic flow through rapidly diverging nozzles, the effects of finite rate kinetics can significantly affect the exit state, and therefore the measured impulse [25], such that the extreme assumptions of either frozen or equilibrium composition may be inadequate.

To evaluate the extent of the effect of chemical kinetics on the impulse in nozzles with dimensions similar to these experiments, the steady-flow conservation equations in one dimension with the species equation are solved [16]. Calculations using STANJAN [26] determine the flow expansion through the nozzle, assuming either equilibrium or frozen composition.

Figure 4 plots the nozzle exit velocity as a function of the environmental pressure. The data points represent the average exit velocity calculated from the measured values of $I_{\mathrm{sp}}$ using Eq. (5). The thick solid line represents the predictions assuming equilibrium composition throughout the nozzle starting from an upstream pressure equal to the state- 3 pressure. The thick dashed line represents the predictions assuming equilibrium composition through the nozzle starting from an average upstream pressure. The two thin dashed lines represent the predictions assuming finite rate chemical kinetics through 8- and 12-deg nozzles, starting from an average upstream pressure.

It is clear that the predictions starting from the average upstream pressure better represent the data. The data points with velocities greater than the steady-flow velocity predictions illustrate the unsteady-flow regime. It is clear that as the environmental pressure decreases, the nozzle shifts from operating in the unsteady-flow regime to the quasi-steady-flow regime. At the lowest environmental pressures, the model overpredicts the data, which can most likely be attributed to the number of assumptions made in representing an inherently unsteady detonation tube with a steady-flow model. It should also be noted that for the relatively short and small divergence nozzles used in this study, an equilibrium composition assumption appears to be valid because the finite rate kinetics do not affect the impulse significantly until much lower environmental pressures used in this study.

\section{C. $I_{\text {sp }}$ Comparisons}

The modeling results of Fig. $\underline{4}$ are plotted in Fig. $\underline{5}$ in terms of $I_{\mathrm{sp}}$ versus the nozzle pressure ratio. The data with nozzles (this report) 


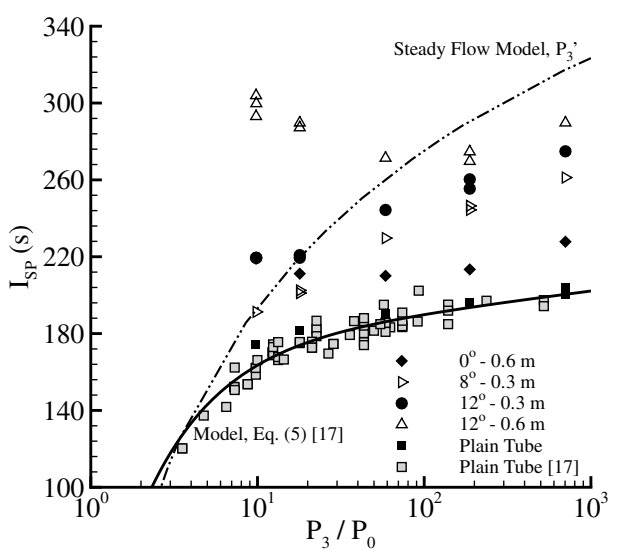

Fig. 5 Specific impulse as a function of the nozzle pressure ratio. The steady-flow predictions based on isentropic expansion from the state-3 pressure $P_{3}$ and the intermediate pressure $P_{3}^{\prime}$ are also plotted.

and impulse data on plain tubes (without nozzles [17]) are also plotted. The data on plain tubes [17] were obtained with ethyleneoxygen mixtures with initial pressures between 30 and $100 \mathrm{kPa}$ in environmental pressures between 1.4 and $100 \mathrm{kPa}$. The thick solid line represents the predictions using the model of Wintenberger et al. [18] modified to account for the increased blowdown time to the lower environmental pressures [17]. The dashed line represents the steady-flow model predictions based on a nozzle inlet pressure of $P_{3}$ that was scaled by $P_{3} / P_{3}$ to plot on the figure in terms of $P_{3} / P_{0}$.

Figure 5 clearly shows that the presence of a nozzle increases the impulse over the baseline case of no attached nozzle. Although the impulse model of Wintenberger et al. [18] modified for application to subatmospheric pressures [17] represents the baseline data well, it is not applicable to tubes with nozzles. Instead, at the largest nozzle pressure ratios, steady-flow predictions from an average intermediate tube pressure represent the data and denote the transition between quasi-steady and unsteady-flow processes that dominate the impulse. The diverging nozzle behavior over the entire range of environmental pressures cannot be represented with a single unifying model, but can be represented at the extremes in which either the quasi-steady or unsteady-flow processes dominate.

This work is applied to single-cycle operation, and so additional factors should be considered when applying these results to multicycle operation. In particular, the nonideal processes of boundary flow separation and nozzle startup time are expected to be minimized, because there is more time during a cycle in which flow exists within the nozzle. Alternatively, the impulse benefit due to the tamper at larger environmental pressures would be expected to suffer from having an initial flow within the nozzle, because the momentum distribution between the tube, exhaust, and tamper gases would be affected.

\section{Unsteady-Flow Regime}

The explosive mass fractions for each nozzle and environmental pressure were given in Table 2 and the data are plotted with the partial-fill model for an asymmetric sandwich $[16,19]$ in Fig. 6 . The experimental data with nozzles are normalized by $I_{\mathrm{sp}}^{\circ}=173 \mathrm{~s}$, which is the experimentally measured value from a fully filled plain tube with a $105-\mu \mathrm{m}$-thick diaphragm and an initial pressure of $80 \mathrm{kPa}$.

The experimental data of Fig. 6 can be divided into three groups. The first group has mass fractions of less than 0.4. These data correspond to the highest environmental pressure and the largest nozzle. The partial-fill model is based on one-dimensional geometries and overpredicts the impulse obtained with a tube and a diverging nozzle. Thus, when the partial-fill effect of the tamper dominates the impulse, a larger increase in impulse is gained with one-dimensional geometries (model predictions) than with a diverging nozzle. For these cases of large nozzles and high environmental pressures, the partial-fill effect is of greatest

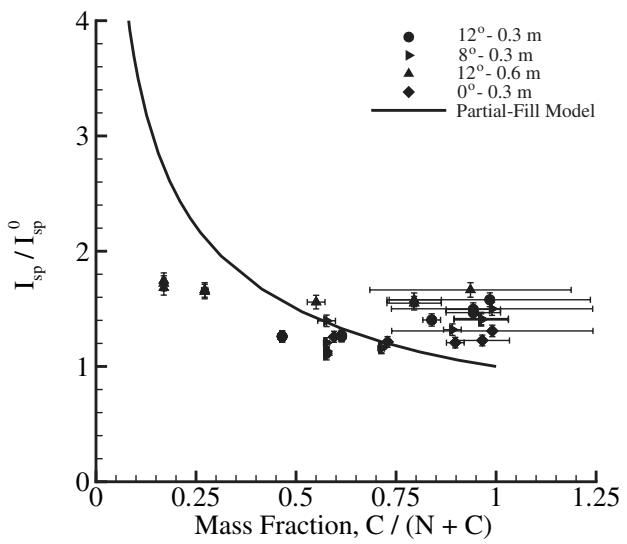

Fig. 6 Normalized specific impulse as a function of the explosive mass fraction. The partial-fill model [16] is plotted with the experimental data for tubes with nozzles.

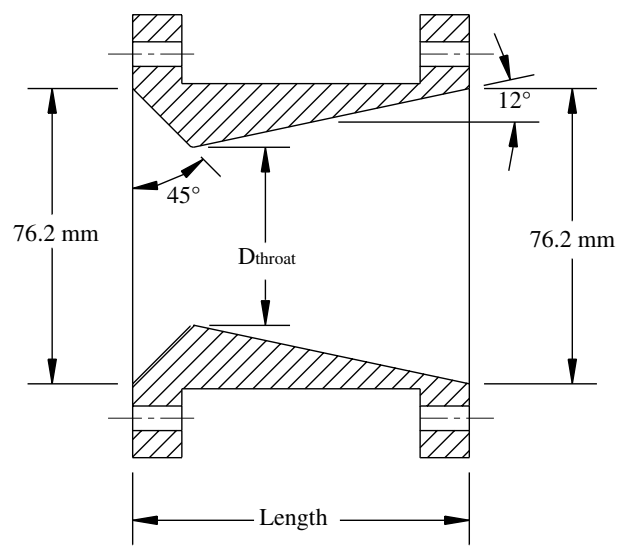

Fig. 7 Illustration of a general converging-diverging throat section located between the exit of the constant-diameter portion of the detonation-tube exit and the inlet of the diverging nozzle. Refer to Table 3 for the exact dimensions.

importance. This effect lessens as the environmental pressure decreases and the explosive mass fraction increases.

For intermediate mass fractions between 0.4 and 0.75 , the partialfill model is in reasonable agreement with the data. These data correspond to the nozzles with the smallest volumes (in which the effect of the divergent shape is minimized) and the larger environmental pressures. The data of the straight extension at the larger environmental pressures are observed to be best predicted by the one-dimensional partial-fill model for this range of explosive mass fractions.

The data at the highest mass fractions, greater than 0.75 , correspond to all of the nozzles and the lowest environmental pressures. It is obvious that the experimental results are uncorrelated with the explosive mass fraction for this situation and the nozzle is operating in the quasi-steady-flow regime. The partial-fill model is not able to model the increased blowdown time and flow expansion that occur within the nozzle.

\section{Converging-Diverging Nozzle Results}

An additional series of tests were conducted by installing a converging-diverging throat section between the constant-diameter portion of the detonation tube and the inlet to the 12-deg diverging nozzles. The inlet and exit diameters of the throat sections, along with the detonation-tube diameter and nozzle inlet diameter, all equaled $76.2 \mathrm{~mm}$. Thus, the diameter continuously decreased from the detonation tube to the throat, followed by a continuous diameter increase from the throat to the nozzle exit. The length of the converging-diverging section varied to yield three different throat areas for a total of six test configurations (see Fig. $\underline{7}$ and Table $\underline{3}$ ). The 
Table 3 Dimensions of the converging-diverging throat sections (refer to Fig. 7 for the corresponding labels)

\begin{tabular}{lccc}
\hline \hline Descriptor & Length, mm & $D_{\text {throat }}, \mathrm{mm}$ & $A_{\text {throat }} / A_{i}$ \\
\hline CD-0.75 & 29.0 & 66.0 & 0.75 \\
CD-0.54 & 57.9 & 55.9 & 0.54 \\
CD-0.36 & 86.9 & 45.7 & 0.36 \\
\hline \hline
\end{tabular}

experimental data appear in Fig. 8 for the 12-deg, 0.3-m nozzle and in Fig. 9 for the 12-deg, 0.6-m nozzle as a function of the environmental pressure.

Analyzing the control volume shown in Fig. 10 for the case with a converging-diverging nozzle requires consideration of the nozzle surfaces that contribute forces in the $x$ direction such as the thrust surface $A_{\mathrm{TS}}$, the converging portion of the nozzle $A_{C}$, and the diverging portion of the nozzle $A_{D}$

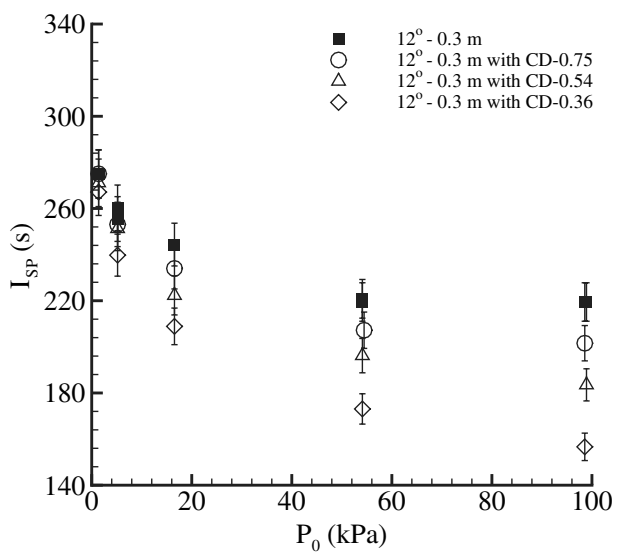

Fig. 8 Specific impulse data for the 12deg, 0.3-m nozzles with converging-diverging sections as a function of the environmental pressure.

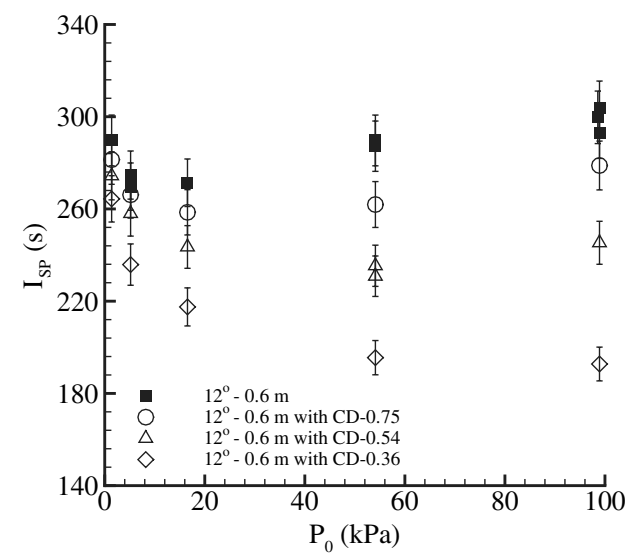

Fig. 9 Specific impulse data for the 12-deg, 0.6-m nozzles with converging-diverging sections as a function of the environmental pressure.

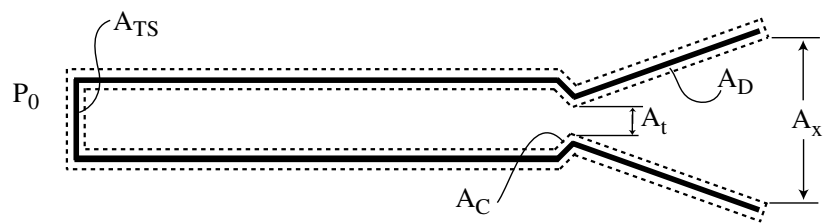

Fig. 10 Control volume for a tube with a converging-diverging nozzle.
The total force on the tube depends not only on the time-varying pressure on the thrust surface, but also on the time-varying pressure on these additional areas. When the environmental pressure is large, the impulse decreases as the throat area decreases. This can be attributed to the presence of large regions of separated flow in the diverging portion of the nozzle such that the force contributions from $A_{D}$ are small. Additionally, decreasing the throat area increases the residence time of the gas within the tube such that heat losses may become significant [27]. An approximation of the loss of impulse expected at atmospheric environmental conditions can be obtained with the impulse model of Wintenberger et al. [18]. A 36\% decrease in the throat area (as is the case for the data of CD-0.36 in Figs. 8 and 9) results in a $36 \%$ impulse loss using $I=K V / U_{\mathrm{CJ}}\left(P_{3}-P_{0}\right)$, where $\bar{V}=A L$, and the state-3 pressure is assumed to equal the pressure on surface $A_{C}$. This predicted $36 \%$ impulse loss is compared with the measured impulse loss of $27 \%$ for the $0.3-\mathrm{m}$-long nozzle and the measured impulse loss of $42 \%$ for the 0.6 -m-long nozzle.

As the environmental pressure decreases, the effect of the converging-diverging restrictions decreases such that at $P_{0}=1.4 \mathrm{kPa}$, each nozzle configuration gives approximately the same value of impulse. In this situation, the large pressure ratio across the nozzle and the resulting quasi-steady nozzle flow dominates the impulse.

\section{Conclusions}

Experiments measuring the single-cycle impulse from detonation tubes with diverging and converging-diverging nozzles were conducted as a function of environmental pressure. Impulses measured from detonation tubes containing diverging nozzles were greater than impulses measured from plain tubes (no nozzle) at all environmental pressures between 100 and $1.4 \mathrm{kPa}$. Depending on the environmental pressure and nozzle shape, the nozzle operated in one of two regimes: the unsteady-flow regime or the quasi-steady-flow regime. In the unsteady-flow regime, the nozzle volume dominates the impulse that was modeled using the partial-fill model. A straight extension is more effective than a diverging nozzle at increasing the impulse for tubes with equivalent explosive mass fractions within the unsteady-flow regime. In the quasi-steady-flow regime, the nozzle area ratio dominates the impulse that was modeled assuming steady flow from an average upstream pressure. A diverging nozzle is more effective than a straight extension at increasing the impulse for tubes with an equivalent explosive mass fraction within the quasi-steadyflow regime. A converging-diverging nozzle was found to be less effective at increasing the impulse than a diverging nozzle at large environmental pressures, due to the flow restriction at the throat. At low environmental pressures, the effect of the throat restriction on impulse was minimized.

\section{Acknowledgment}

This work was supported by the Office of Naval Research Multidisciplinary University Research Initiative, Multidisciplinary Study of Pulse Detonation Engine (N00014-02-1-0589), and General Electric contract GE-PO A02 81655 under DABT-63-00001 .

\section{References}

[1] Cambier, J.-L., and Tegner, J. K., "Strategies for Pulsed Detonation Engine Performance Optimization," Journal of Propulsion and Power, Vol. 14, No. 4, July-Aug. 1998, pp. 489-498.

[2] Eidelman, S., and Yang, X., "Analysis of the Pulse Detonation Engine Efficiency," 34th AIAA/ASME/SAE/ASEE Joint Propulsion Conference and Exhibit, Cleveland, OH, AIAA Paper 1998-3877, July 1998.

[3] Guzik, S. M., Harris, P. G., and Champlain, A. D., "An Investigation of Pulse Detonation Engine Configurations using the Method of Characteristics," 38th AIAA/ASME/SAE/ASEE Joint Propulsion Conference and Exhibit, Indianapolis, IN, AIAA Paper 2002-4066, July 2002.

[4] Wu, Y., Ma, F., and Yang, V., "System Performance and Thermodynamic Cycle Analysis of Air-Breathing Pulse Detonation 
Engines," Journal of Propulsion and Power, Vol. 19, No. 4, JulyAug. 2003, pp. 556-567.

[5] Morris, C. I., "Numerical Modeling of Single-Pulse Gasdynamics and Performance of Pulse Detonation Rocket Engines," Journal of Propulsion and Power, Vol. 21, No. 3, May-June 2005, pp. 527538.

[6] He, X., and Karagozian, A. R., "Pulse-Detonation-Engine Simulations with Alternative Geometries and Reaction Kinetics," Journal of Propulsion and Power, Vol. 22, No. 4, July-Aug. 2006, pp. 852861.

[7] Allgood, D., Gutmark, E., Hoke, J., Bradley, R., and Schauer, F., "Performance Measurements of Multicycle Pulse-Detonation-Engine Exhaust Nozzles," Journal of Propulsion and Power, Vol. 22, No. 1, Jan.-Feb. 2006, pp. 70-77.

[8] Cooper, M., Jackson, S., Austin, J. M., Wintenberger, E., and Shepherd, J. E., "Direct Experimental Impulse Measurements for Detonations and Deflagrations," Journal of Propulsion and Power, Vol. 18, No. 5, Sept.-Oct. 2002, pp. 1033-1041.

[9] Falempin, F., Bouchaud, D., Forrat, B., Desbordes, D., and Daniau, E., "Pulsed Detonation Engine Possible Application to Low Cost Tactical Missile and to Space Launcher," 37th AIAA/ASME/SAE/ASEE Joint Propulsion Conference and Exhibit, Salt Lake City, UT, AIAA Paper 2001-3815, July 2001.

[10] Owens, Z. C., Mattison, D. W., Barbour, E. A., Morris, C. I., and Hanson, R. K., "Flowfield Characterization and Simulation Validation of Multiple-Geometry PDEs Using Cesium-Based Velocimetry, Part 2," Proceedings of the Combustion Institute, Vol. 30, Combustion Inst., Pittsburgh, PA, 2005, pp. 2791-2798.

[11] Kailasanath, K., "Recent Developments in the Research on Pulse Detonation Engines," AIAA Journal, Vol. 41, No. 2, Feb. 2003, pp. $145-159$.

[12] Owens, Z. C., and Hanson, R. K., "Unsteady Nozzle Design for Pulse Detonation Engines," 41st AIAA/ASME/SAE/ASEE Joint Propulsion Conference and Exhibit, Tucson, AZ, AIAA Paper 2005-3649, July 2005.

[13] Tangirala, V. E., and Dean, A. J., "Performance Estimations of a Pulse Detonation Engine with Exit Nozzle," 42nd AIAA/ASME/SAE/ASEE Joint Propulsion Conference and Exhibit, Sacramento, CA, AIAA Paper 2006-4792, July 2006.

[14] Ma, F., Choi, J.-Y., and Yang, V., "Propulsive Performance of Airbreathing Pulse Detonation Engines," Journal of Propulsion and Power, Vol. 22, No. 6, Nov.-Dec. 2006, pp. 1188-1203. doi: $10.2514 / 1.21755$
[15] Harris, P. G., Stowe, R. A., Ripley, R. C., and Guzik, S. M., "Pulse Detonation Engine as a Ramjet Replacement," Journal of Propulsion and Power, Vol. 22, No. 2, Mar.-Apr. 2006, pp. 462-473.

[16] Cooper, M. A., "Impulse Generation by Detonation Tubes," Ph.D. Thesis, California Inst. of Technology, Pasadena, CA, 2004.

[17] Cooper, M., and Shepherd, J., "Detonation Tube Impulse in Subatmospheric Environments," Journal of Propulsion and Power, Vol. 22, No. 4, July-Aug. 2006, pp. 845-851.

[18] Wintenberger, E., Austin, J. M., Cooper, M., Jackson, S., and Shepherd, J. E., "Analytical Model for the Impulse of a Single-Cycle Pulse Detonation Tube," Journal of Propulsion and Power, Vol. 19, No. 1, Jan.-Feb. 2003, pp. 22-38.

[19] Kennedy, J. E., "The Gurney Model of Explosive Output for Driving Metal," Explosive Effects and Applications, edited by J. A. Zukas and W. P. Walters, Springer, New York, 1998, pp. 221-257, Chap. 7.

[20] Sutton, G. P., Rocket Propulsion Elements, 6th ed., Wiley, New York, 1992, pp. 41-68.

[21] Amann, H. O., "Experimental Study of the Starting Process in a Reflection Nozzle," Physics of Fluids, Vol. 12, No. 5, May 1969, pp. I150-I-153. doi:10.1063/1.1692597

[22] Smith, C. E., "The Starting Process in a Hypersonic Nozzle," Journal of Fluid Mechanics, Vol. 24, No. 4, 1966, pp. 625-640. doi:10.1017/S0022112066000880

[23] Saito, T., and Takayama, K., "Numerical Simulations of Nozzle Starting Process," Shock Waves, Vol. 9, No. 2, Apr. 1999, pp. 73-79. doi:10.1007/s001930050141

[24] Chen, C. L., Chakravarthy, S. R., and Hung, C. M., "Numerical Investigation of Separated Nozzle Flows," AIAA Journal, Vol. 32, No. 9, Sept. 1994, pp. 1836-1843.

[25] Scofield, M. P., and Hoffman, J. D., "Maximum Thrust Nozzles for Nonequilibrium Simple Dissociating Gas Flows," AIAA Journal, Vol. 9, No. 9, 1971, pp. 1824-1832.

[26] Reynolds, W. C., "The Element Potential Method for Chemical Equilibrium Analysis: Implementation in the Interactive Program STANJAN, Version 3," Department of Mechanical Engineering, Stanford Univ., Stanford, CA, Jan. 1986

[27] Radulescu, M. I., and Hanson, R. K., "Effect of Heat Loss on PulseDetonation-Engine Flow Fields and Performance," Journal of Propulsion and Power, Vol. 21, No. 2, Mar.-Apr. 2005, pp. 274-285.

J. Powers Associate Editor 